\title{
Relevancia de la anatomía humana en el ejercicio de la medicina de asistencia primaria y en el estudio de las asignaturas de segundo ciclo de la liconciatura en medicina
}

\author{
Blanca Mompeó y Lilián Pérez \\ Departamento de Morfología. U niversidad de Las Palmas de Gran Canaria
}

El objetivo de este trabajo fue con ocer la relevancia $y$ lanecesidad que deconocimientos en Anatomía macroscópica tenían los médicos deasistencia primaria y los alumnos del segundo ciclo de la licenciatu ra en Medicina. También nos interesó saber cual era su opin ión sobre esta discip lina del curriculum médico. Para ello, médicos de asistencia primaria de los Centros de Salud de la provincia de las Palmas y alumnos de $6^{\circ}$ curso de la Licenciatura en Medicina de la U.L.P.G.C., cumplimentaron un cuestionario en el que se valoraron los aspectos referidos. Ambos grupos consideraron que la An atomía era fundamental para la exploración física y para la in terpretación de técnicas de imagen. Los conocimientos más necesarios a la hora de su aplicación a la práctica y al estudio de las asignat uras clínicas fueron los de sistema nervios o y aparato locomotor: Modificarían la docencia que recibieron, in crement an do el número de clases prácticas y dándole un mayo $r$ enfo que clínico. Los tres objetivos fundamentales de aprendizaje propu estos por los profesionales en ejercicio fu eron: 1. Con ocer las estru cturas an atómicas y relaciones ent re ellas 2 . Reco nocer es truct uras anató micas mediante técn icas de imagen y 3. Ser capaz de des cribir las bas es anatómicas de la patología.

Palabras clave:

Anatomía macroscópica, Relevancia clínica, Curriculum médico, Docencia de la Anatomía Humana

Correspondencia:

Blanca Mompeó Cor redera

Departamento de Morfología

Universidad de Las Palmas de Gran Canaria (U.L.P.G.C.)

Edificio de Ciencias de la Salud

Dr. Pasteur s/n Trasera del Hospital Insular

35016 Las Palmas de Gran Canaria

Telefono: 928453414 - Fax: 928453420

Correo electrónico: bmompeo@dmor.ulpgc.es
Relevance of Gross Human Anatomy in health primary care and in clinical dis ciplines of medical studie s.

The aim of this work was to high light the relevance and neces sity about gross anato my knowledge had on family physicians and clinical-courses medical students. We also wanted to know their suggestions about the discipline Human Anatomy in Medical studies. To obtain our objective, physicians of Health Centres in Las Palmas and last-year medical students of U.L.P.G.C. were asked to fill a questionnaire. In this questionnaire the referred items were considered. The data obtained showed that both groups thought that Gross Anatomy was fundamental in the physical exploration and the image techniques int erpretation. They considered that anatomical knowledge more necessary for clinical practise and clinical studies were nervous and locomotion systems. They would change the way they practical were taught in: Increased number of practical lessons, and increased clinical view. The main ly learning proposed objectives w ere: 1. To Know anatomic stru ctures and relatio nsh ips between them. 2. Iden tify anatomic structures by image techniques. 3 . To be able to des cribe the anatomical bases of pathology.

Key words:

Gross anatomy, Clinical relevance, Medical curriculum, Teaching of Anatomy

\section{INTRODUCCIÓN}

Actualmente se considera que son las necesidades de la sociedad y no otro tipo de intereses las que deben determinar los fines de la educación. Esta concepción obliga a la institución universitaria a ofrecer programas y curricula basados en competencias profesionales. Este tipo de enseñanza es parti- 
cularmente necesaria en las titulaciones sanitarias y en este sentido se manifiesta la OMS1.

Aunque parece existir acuerdo general en que la disciplina de Anatomía Humana es uno de los pilares fundamentales en los que se sustentan las diencias médicas, algunas experi encias han demostrado que a la hora de establ ecer objetivos de aprendizaje estos difieren considerablemente según sean sugeridos por un profesor de Anatomía o por un profesional dedicado a la práctica dínica ${ }^{2}$. Consideramos por tanto que la investigación docente encaminada a definir metas adecuadas de aprendizaje pasa necesariamente por la entrevista a los profesionales dínicos.

Las entrevistas a profesionales en ejercicio ayudan a conocer con precisión cuales son las funciones que estos deben desempeñar en el trabajo diario, así como el curriculum nulo o necesidades de formación previa que el médico detecta en su práctica diaria ${ }^{3,4}$. Por ello, las respuestas obtenidas por este método son de máxima utilidad para concebir objetivos de aprendizaje.

Otra opinión importante es la de los alumnos, tanto en lo relativo a la aplicabilidad de los conocimientos en Anatomía Humana a otras disciplinas, como en su satisfacción personal con la docencia recibida.

Si bien las encuestas a los alumnos no se consideran adecuadas para la elaboración de objetivos de aprendizaje, son útiles a la hora de valorar los objetivos puente, el curriculum nulo, la sobrecarga de contenidos, así como la actividad docente del profesorado 5 . La regularidad en la realización de las mismas conlleva una mejora en la docencia ya que suministra bases e ideas para la realización de posibles reformas ${ }^{6}$.

Los trabajos en investigación docente, destinados a una valoración de los estudios de Anatomía Humana del currículum médico, por parte de estudiantes y profesionales clínicos, son escasos. En el aspecto concreto que nos ocupa tenemos referencia de un trabajo basado en la valoración que hicieron los alumnos del último curso de la licenciatura en Medicina, y de un segundo estudio en el que se recogían las opiniones de especialistas tras la finalización de su residencia hospitalaria. Ambos trabajos estaban en relación a la anatomía macroscópica que los entrevistados habían estudiado en la universidad de Hamburgo ${ }^{7,8}$. No tenemos referencias de que este tipo de estudio se halla realizado en ninguna de las comunidades de nuestro país.

Nuestra intención fue averiguar cuales eran las necesidades del médico de asistencia primaria y del estudiante en el segundo ciclo de la licenciatura en medicina respecto a anatomía macroscópica. Por otra parte quisimos también conocer cual era su opinión en lo que respecta a la Anatomía Humana como disciplina en general, sus diferentes aspectos y su docencia.

Todo ello con el objetivo final de iniciar una línea de investigación docente que nos permita concebir y proponer programas más pertinentes y adecuados a las necesidades de nuestra sociedad.

\section{MATERIAL Y MÉTO DO:}

El estudio se desarrolló en dos poblaciones:

1- Médicos pertenecientes a los equipos de asistencia primaria del Servicio Canario de Salud de la provincia de Las Palmas. Estos profesionales desarrollaban su labor profesional en diferentes Centros de Salud de la isla de Gran Canaria.

2- Estudiantes matriculados en las asignaturas troncales de sexto curso de la licenciatura en Medicina de la Universidad de Las Palmas de Gran Canaria durante los cursos académicos 1998-99 $(n=13)$ y $2000-2001(n=11)$. Los alumnos participantes fueron aquellos que con mayor regularidad acudían a las clases teóricas y que constituyeron un $26 \%$ y $25 \%$ respectivamente de los matriculados. Los dos grupos de alumnos cursaron sus estudios de Anatomía Humana durante los dos primeros años de la Licenciatura. Para la mayoría de ellos correspondió a los cursos académicos 93/94, 94/95 y 95/96,96/97 respectivamente. El primer grupo cursó sus estudios según el plan antiguo, mientras que el segundo lo hizo según el nuevo plan (BOE 12/09/94). Los profesores responsables de las asignaturas de Anatomía Humana durante los años referidos fueron diferentes. Los autores del trabajo contribuimos a la docencia de los dos grupos y fuimos los responsables de los contenidos de esplacnología y sistema nervioso durante el curso académico 1996/97.

Un total de 225 cuestionarios (anexol) fueron distribuidos en 24 Centros de Salud de la provincia de Las Palmas. El cuestionario fue de carácter anónimo y constó de 24 preguntas, 21 de las cuales fueron de tipo cerrado y tres de carácter abierto. En 5 preguntas requerimos una puntuación de 1-5 considerada de menor a mayor calificación en la valoración de cada respuesta. Las preguntas abiertas fueron planteadas para que los participantes pudieran realizar comentarios o sugerencias libremente.

La final idad de la encuesta fue conocer cual era la consideración de estos profesionales respecto a la 
TABLA 1 Aspectos considerados en los cuestionarios
Alumnos
a) Filiación
b) Consideraciones sobre las asignaturas básicas de la licenciatura en medicina.
c) Valoración de la utilidad de los conocimientos anatómicos en las asignaturas clínicas.
d) Impresión personal sobre la docencia de la Anatomía Humana

\section{Profesionales en ejercicio}

a) Filiación

b) Consideraciones sobre asignaturas básicas de la licenciatura en Medicina

c) Consideraciones sobre la Anatomía Humana como asignatura en particular

d) Relaciones entre el ejercicio profesional y los conocimientos anatómicos

f) Docencia de la Anatomía

1. Valoración sobre la docencia en anatomía recibida

2. Objetivos propuestos a alcanzar tras el desarrollo del programa de Anatomía

asignatura Anatomía Humana del currículum médico y la relevancia de esta asignatura en relación con el ejercicio profesional. También se intentó averiguar cual era su opinión, así comosus suger encias en relación con la docencia de la disciplina. Los diferentes aspectos estudiados se recogen en la tabla 1.

A los alumnos se les distribuyó un cuestionario, también de carácter anónimo, para ser completado voluntariamente Las encuestas les fueron entregadas a un al umno colaborador quien las repartió entre sus compañeros, sin que hubiesen sido convocados previamente. Les explicó lo que se pretendía y les pidió que las rellenar an con sinceridad. Los alumnos quelo desearon contestaron las preguntas en el aul a sin la presencia de profesor. El auestionario (anexo2) constó de 20 preguntas, 16 de tipo cerrado y cuatro de carácter abierto cuya finalidad fue conocer la opinión que el alumno tenía sobre los conocimientos anatómicos que recibió y si estos le fueron deutilidad en el estudio de las asignaturas dínicas (tabla 1).

De los resultados obtenidos en las preguntas en las que se requería valoración numérica se obtuvo la media y desviación típica. Los datos fueron analizados, si era el caso, según las variables de: año de finalización de la licenciatura, titulación y situación laboral.

Para el análisis comparativo de los diferentes grupos se estudiaron las varianzas y la diferencia de medias para muestras independientes. Los grupos fueron comparados dos a dos. EI paquete estadístico utilizado fue el SPSS.

\section{RESULTADOS}

\section{Médicos de equipos de atención primaria}

Nos fueron remitidos 88 cuestionarios, que corresponden a un 39,6\% del total de los repartidos.

Un $76 \%$ de los facultativos estaba contratado por el Servicio Canario de Salud, mientras que un $22,7 \%$ era titular de la plaza. Respecto al sexo el $52.3 \%$ fueron varones y el $45.5 \%$ mujeres.

Un $2 \%$ de los que contestaron la encuesta finalizó sus estudios antes de 1980 , un $44,3 \%$ lo hizo entrelos años $80-84$, un $12,5 \%$ entreel $85-89$ y solo $1.1 \%$ lo hizo después de 1990.

A la petición de que valoraran las asignaturas básicas en relación con la importancia que estas habían tenido en el desarrollo del ejercicio profesional, la Anatomía, como muestra la figura 1, ocupó el tercer lugar después de la Farmacología y la Fisiología.

\section{Relevancia y necesidades de conocimientos de Anatomía macroscópica}

En respuesta a las preguntas referidas a la aplicación de los conocimientos anatómicos a diversas procedimientos en la actividad profesional; Un 73\% consideró que la Anatomía era fundamental en la exploración física de los pacientes, un $50 \%$ que lo era en la interpretación de las técnicas de imagen, un 34\% en la interpretación de la sintomatología y solo un $15 \%$ la consideró fundamental en los procedimientos terapéuticos.

La Anatomía fue considerada de mayor impor tancia en la exploración física, por los especialistas en medidina familiar que por los generalistas $(P=0.019)$.

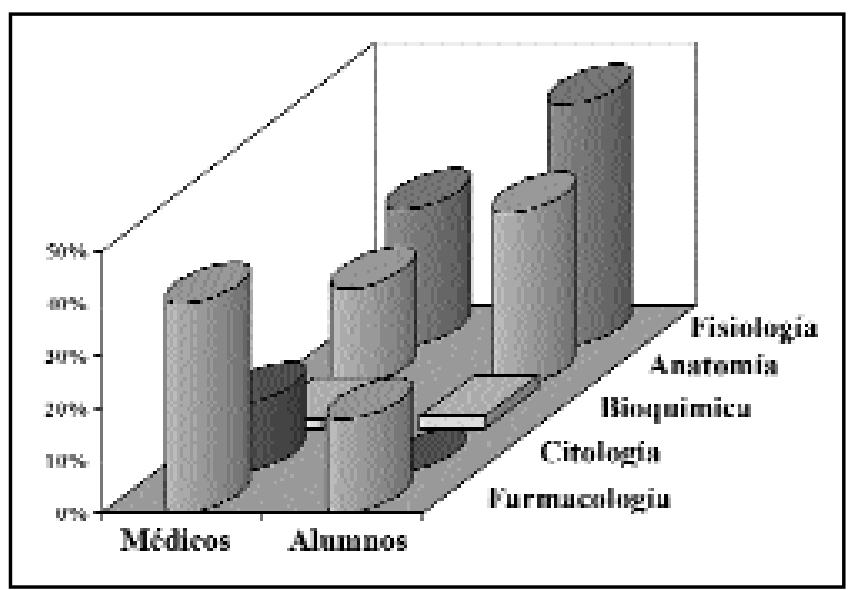

Fig.1: Valoración de las asignaturas básicas según su relevancia en la clínica 
TABLA 2. Valoración de diferentes aspectos de la Anatomía.

TITULACIÓN

Generalistas

Especialistas

M.Familia

Otros Especialistas

AÑO DE FINALIZACIÓN LICENCIATURA

Antes 1980

$1980 / 84$

$1985 / 89$

$1990 / 95$

Después de 1995

SITUACIÓN LABORAL

Titulares

Contratados

\begin{tabular}{|cccccc|} 
Funcional & Imagen & Topográfica & Superficie & Descriptiva & Embriologia \\
\hline $3,6 \pm 1,3$ & $4 \pm 1,1$ & $3,5 \pm 1,3$ & $3,3 \pm 1,4$ & $3,2 \pm 1,3$ & $1,6 \pm 1,2$ \\
& & & & & \\
$4,3 \pm 0,9^{a}$ & $4,1 \pm 0,7$ & $3,5 \pm 1$ & $3,2 \pm 1,1$ & $3,8 \pm 0,9$ & $1,9 \pm 1,2$ \\
$3,3 \pm 1,1$ & $4,1 \pm 0,9$ & $4,3 \pm 0,7$ & $3 \pm 1,9$ & $3,4 \pm 1,4$ & $1,8 \pm 1,3$ \\
& & & & \\
& & & & & \\
$3,6 \pm 1,3$ & $3,8 \pm 1$ & $4,3 \pm 0,9$ & $3,2 \pm 1,6$ & $3,8 \pm 1,1^{\circ}$ & $1,8 \pm 1,1$ \\
$3,9 \pm 1,2$ & $3,9 \pm 0,9$ & $3,2 \pm 1,2$ & $3,3 \pm 1,2$ & $2,8 \pm 1,3$ & $1,5 \pm 1,4$ \\
$4 \pm 1,2$ & $4,5 \pm 0,7^{b}$ & $4 \pm 0,9^{c}$ & $3,4 \pm 1,4$ & $3,5 \pm 1,1$ & $2,3 \pm 1,3^{\mathrm{d}}$ \\
$3 \pm 0$ & $3,8 \pm 1,1$ & $3,7 \pm 0,7$ & $3,1 \pm 1,3$ & $3,1 \pm 1,1$ & $1,5 \pm 1,3$ \\
& $4 \pm 0$ & $5 \pm 0$ & $4 \pm 0$ & $3 \pm 0$ & $2 \pm 0$ \\
$3,9 \pm 1,2$ & $3,9 \pm 1$ & $4,1 \pm 0,9$ & $3,3 \pm 1,6$ & $3,6 \pm 1,3$ & $1,8 \pm 1,3$ \\
$3,7 \pm 1,2$ & $4,1 \pm 0,9$ & $3,6 \pm 1,2$ & $3,4 \pm 1,3$ & $3,2 \pm 1,2$ & $1,9 \pm 1,1$ \\
\hline
\end{tabular}

a. Diferencia en valoración entre médicos de familia y otros especialistas $P=0,016$, entre médicos de familia y generalistas $P=0,025$

b. Diferencia entre los que finalizaron en los años 1985-89 y aquellos que lo hicieron entre 1980-84, $P=0,018$

c. Diferencia entre los años $1985-89$ y $1980-84, \mathrm{P}=0,025$.

d. Diferencia entre los años 1985-89 y 1980-84, $\mathrm{P}=0,033$.

e. Valoración de la anatomía descriptiva antes de 1980 y entre $1980-84, \mathrm{P}=0,04$.

TABLA 3. Valoración de la Anatomía según su aplicabilidad a la clínica

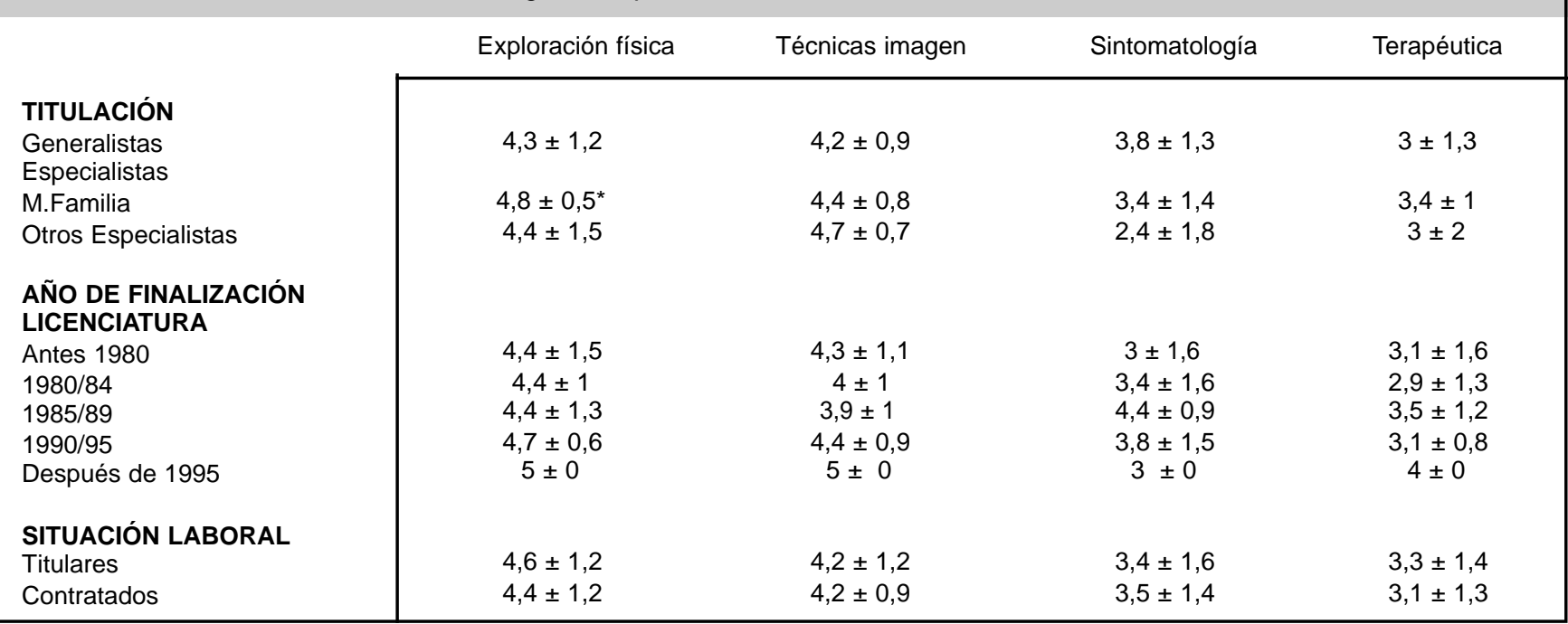

* Diferencia de valoración de los especialistas en medicina de familia con otros especialistas $P=0,016$, con los generalistas $P=0,025$

Nose observaron difer encias de valoración en relación con el año de finalización dela licenciatura, ni en relación con la situación laboral del entrevistado.

Respecto a la relevancia para su labor profesional, los diferentes aspectos de la Anatomía Humana fueron clasificados en el siguiente orden: anatomía por la imagen, seguida de anatomía funcional, anatomía topográfica y anatomía de superficie, ocupando la anatomía descriptiva y la embriología los últimos lugares.
Esta valoración mostró ciertas diferencias según el año de finalización de la licenciatura. En este sentido se observó una mejor valoración de la anatomía topográfica $(P=0,025)$, la anatomía por la imagen $(P=0,018)$ y la embriología $(P=0,033)$ por aquellos profesionales que terminaron su licenciatura entre los años 85-89 que por los que lo hicieron entre 1980 y 1984.

También se observó difer encia entre la consideración de la anatomía descriptiva y topográfica con una 
menor valoración por parte de los licenciados que terminaron entre los años 1980-84 que por los que lo hicieron antes de $1980(P=0,04)$.

Según la titulación, los médicos de familia valoraron más positivamente la anatomía funcional que otros especialistas $(P=0,016)$ y que los generalistas $(P=0,025)$. No se observaron diferencias con relación a la situación laboral.

Las medias y desviaciones típicas obtenidas de los diferentes grupos estudiados se muestran en las tablas 2 y 3.

Mediante dos preguntas se trató de valorar la importancia que para los profesionales de la medicina tenía la anatomía por la imagen. La primera de ellas estaba dirigida a conocer que tipo de prueba diagnóstica basada en la imagen era la que utilizaban con mayor frecuencia. El $87.5 \%$ refirió que empleaba radiología simple frecuentemente, la radidogía con contraste era utilizada de forma habitual solo por un $3,4 \%$, el TAC y la RMN lo eran por un $1,1 \%$ y las ecografías por un $17 \%$.

Respecto a la relevancia que para ellos tenía la anatomía por la imagen en los programas de anatomía, el 89,9\% consideró que era de máxima importancia, mientras que sólo un 2,3\% la consideró poco importante Observamos que el grupo de profesionales que mejor valoróla anatomía por la imagen fue el quefinalizó su licenciatura entre los años 1985/89.

Con respecto a la anatomía de superficie, un $9 \%$ respondió que no servía para nada, mientras que un 83\% opinó que era fundamental o necesaria.

Un 40,9\% repasa sus conocimientos anatómicos cada seis meses, mientras que un $13 \%$ lo hace aproximadamente una vez al mes y solo $2,3 \%$ no lo hace nunca.

U n 88,6\% de los entrevistados consider a de interés la realización de cursos de postgrado en anatomía.

Los profesionales clasificaron, por orden de frecuencia, la necesidad de repasar los conocimientos anatómicos de:

Sistema nervioso, segui do de aparato locomotor en primer y segundo lugar respectivamente, cardiovascular y respiratorio en tercero, y órganos de los sentidos urogenital y digestivo en último lugar. Respecto a la necesidad de conocer las bases anatómicas a la hora de estudi ar a un paciente, consideran queson muy importantes cuan do se trata de un enfermo con problemas trau matológicos un $71,6 \%$ y neurológicos un 53,4\%, seguidos en importancia los problemas cardio-vasculares con un $28 \%$. Fue considerada de menor interés en el estudio del resto de los procesos patológicos.

\section{Opiniones sobre la docencia de la Anatomía Humana}

Con respecto a la docencia de la Anatomía Humana planteamos tres preguntas. Estas se referian a la ubicación temporal de la asignatura, los objetivos que los alumnos deberían alcanzar, así como su impresión personal sobre la Anatomía que recibieron durante su formación.

Respecto a la conveniencia de la ubicación de la Anatomía Humana en el primer o segundo cido de la licenciatura, un $83 \%$ consideró que sería de interés la enseñanza de conocimientos generales de anatomía macroscópica durante el primer cido de la licenciatura y de una anatomía aplicada durante el segundo ciclo. Un $11,4 \%$ consideró que debería seguir impartiéndose únicamente en primer cido.

Tres fueron los objetivos fundamentales propuestos por los médicos de asistencia primaria a alcanzar tras el desarrollo del programa de Anatomía Humana:

1. Ser capaz de describir las bases anatómicas de la patología

2. Conocer las estructuras anatómicas y las relaciones entre ellas.

3. Ser capaz de reconocer estructuras anatómicas mediante técnicas de imagen.

A la pregunta de qué modificarían y qué conservarían de la enseñanza en Anatomía Humana que recibieron, no contestó un 31,8\%, un 7,9\% declaró estar conforme con la docencia que recibió y un 5,68\% manifestó estar descontento con la misma.

EI resto de los profesionales consideró que no fue la más adecuada y los aspectos a modificar señalados con mayor frecuencia fueron los siguientes:

a) La relación clases teóricas/clases prácticas incrementando el número de estas últimas tanto con técnicas de imagen como con disección $(68,5 \%)$,

b) La orientación de la asignatura, aplicándola a la práctica clínica y al ser humano vivo, el (36,08\%).

c) La manera de impartir la asignatura, haciéndolo de forma más amena, con una mayor participación por parte del alumno (18,04\%).

Cuando se les pidió que definieran el concepto de "Anatomía Clínica" y que expresaran su opinión sobre la misma, lo hizo un $51 \%$, mientras que no respondió el 49\%. Recogemos en la Tabla 5 algunas de esas definiciones y opiniones.

\section{Grupo de alumnos de sexto curso de la Licenciatura en Medicina}

El cuestionario fue respondido por un $54 \%$ de varones y un $46 \%$ de mujeres. 


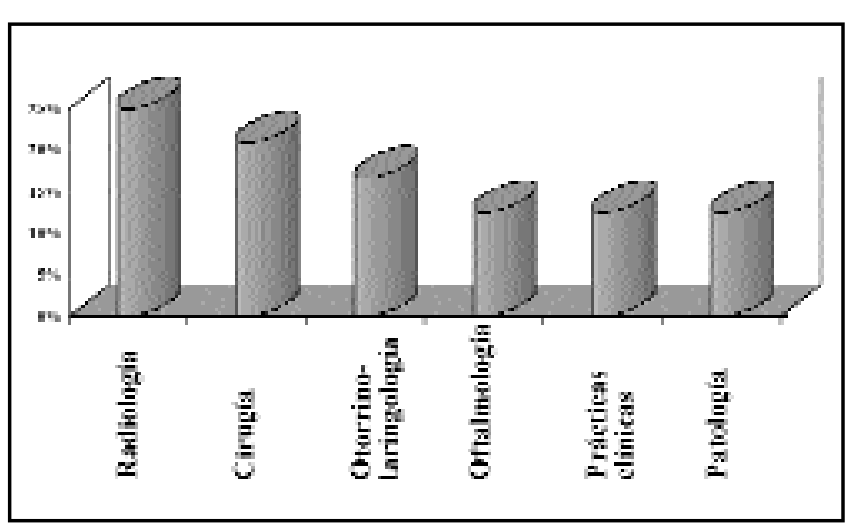

Fig.2: Relación de la Anatomía Humana con las asignaturas clínicas

Las tres asignaturas básicas que consideraron más relacionadas con las dínicas fueron; la Fisiología que ocupó el primer lugar, seguida por la Anatomía Humana y la Farmacología en segundo y tercer lugar respectivamente (fig.1).

\section{Relevancia y necesidades de conocimientos de Anatomía macroscópica}

El 90,9\% de los alumnos consideró que los conocimientos anatómicos son relevantes en la exploración física y la interpretación de las técnicas de imagen. La consideraron de menor importancia en la interpretación de los síntomas y en la terapéutica.

El $41,6 \%$ de los alumnos estimaron fundamental la anatomía por la imagen, opinando el 79,2\% que la misma debería ocupar un lugar importante dentro de los programas de anatomía. Un 37,5\% adjudicó una mejor valoración a la anatomía funcional. En general consideraron de poca relevancia los conocimientos adquiridos en Embriología.

Con respecto a la anatomía desuper ficie, el 91,7\% de los alumnos pensó que era fundamental o necesaria. El 8,3\% por el contrario la consideró poco úti l.

El 16,6\% repasa sus conocimientos anatómicos una vez al mes, e $25 \%$ una vez cada 6 meses, y un $4 \%$ no lo hacenunca.

Los alumnos refirieron que para el estudio de las asignaturas dínicas habían necesitado repasar sus conocimientos de anatomía del sistema nervioso, seguido de aparato locomotor y con menor frecuencia los conocimientos de órganos de los sentidos, aparato cardiovascular, sistema urogenital y aparato digestivo.

A la hora de estudiar un paciente los alumnos necesitaron tener conocimientos anatómicos sobre todo si se trataba de un paciente con patología neurológica el $62,5 \%$, traumatológica, el 58,3 \% y oftalmológica el $20,8 \%$.
Respecto a la necesidad de tener conocimientos anatómicos para el estudio de otras asignaturas, el $41,6 \%$ de los alumnos consideró que estos les fueron útiles sobre todo en la Radiología General. Un $25 \%$ los consideró de más utilidad en la Cirugía general, y un $20,8 \%$ en Otorrinolaringología (fig.2).

\section{Opiniones sobre la docencia de Anatomía Humana.}

EI 45,8 \% consideró aceptable el hecho de que la Anatomía Humana sea impartida en el primer ciclo de la licenciatura, mientras que $41,6 \%$ consideró de mayor utilidad impartir una anatomía general durante el primer ciclo de la licenciatura y una anatomía aplicada durante el segundo ciclo de la misma. Un 12,5\% opinó que se debería disminuir el número de horas dedicadas a esta asignatura.

A la pregunta de qué modificarían de la Anatomía que habían recibido los alumnos contestaron que:

1. Disminuirían el número de clases magistrales, los datos a aprender de memoria, así como los datos poco útiles en la práctica clínica.

2. Incrementarían el número de dases prácticas incluyendo más horas de disección técnicas de imagen y resolución de problemas.

Las definiciones de Anatomía dínica expresadas por los alumnos se recogen en la tabla 5.

\section{Estudio comparativo de ambos grupos}

Varias de las preguntas de los cuestionarios fueron similares o coincidentes para ambos grupos. Las valoraciones obtenidas en las mismas se recogen en la tabla 4.

\section{Relevancia y necesidades de conocimientos de anatomía macroscópica}

Los al umnos consideraron que la Anatomía Humana era la segunda asignatura básica con mayor aplicación clínica y los médicos de asistencia primaria, la tercera. No hubo diferencia significativa en la valoración general dada por alumnos y profesionales a la Anatomía $(P=0,36)$, a la Fisiología $(P=1)$, ni a la Citología $(P=0,1)$. Sí hubo variaciones en la valoración de la Bioquímica y la F armacología, con una mejor puntuación por parte de los profesionales ( $P=0,01$ y $P=0,001$ respectivamente).

Los dos grupos coincidieron en dasificar la exploración física en primer lugar y la interpretación de técnicas de imagen en segundo, como las actividades clínicas en las que la Anatomía es fundamental. Situando posteriormente la interpretación de la sintomatología y los procedimientos terapéuticos. No 
TABLA 4. Valoración atribuida por alumnos y profesionales

Aplicabilidad Clínica de la Anatomía Humana

\begin{tabular}{l|ccccc} 
& Anatomía & Fisiología & Citología & Bioquímica $^{a}$ & Farmacología $^{b}$ \\
\cline { 2 - 6 } Médicos & $3,6 \pm 1$ & $4,1 \pm 1,1$ & $2,2 \pm 1,2$ & $2,5 \pm 1,1$ & $4,3 \pm 0,9$ \\
Alumnos & $3,8 \pm 1$ & $4,1 \pm 1$ & $2,5 \pm 0,8$ & $1,8 \pm 0,9$ & $3,3 \pm 1,1$
\end{tabular}

Actividades clínicas donde la anatomía es fundamental

\begin{tabular}{ccccc} 
& Exploración & Imagen & Sintomatología & Terapéutica \\
\cline { 2 - 5 } Médicos & $4,5 \pm 0,7$ & $4,2 \pm 0,9$ & $3,7 \pm 1,2$ & $3,1 \pm 1,23$ \\
Alumnos & $4,5 \pm 1$ & $4,5 \pm 0,8$ & $3,6 \pm 0,8$ & $3,2 \pm 1,25$
\end{tabular}

Aspectos de la anatomía con mayor aplicabilidad

\begin{tabular}{lcccccc|} 
& Descriptiva & Funcional & Superficie & Imagen & Topogràfica & Embriología \\
\cline { 2 - 7 } Médicos & $3,3 \pm 1,1$ & $3,7 \pm 1,2$ & $3,3 \pm 1,3$ & $4 \pm 0,9$ & $3.7 \pm 1,13$ & $2 \pm 1,1$ \\
Alumnos & $3,2 \pm 1,2$ & $3,9 \pm 1,2$ & $3,8 \pm 1,1$ & $4 \pm 0,9$ & $4 \pm 0,7$ & $1,9 \pm 1$
\end{tabular}

Necesidad de recordar conocimientos

\begin{tabular}{lcccccc|} 
& Cardio-vascular & Digestivo & Locomotor & Uro-genital & Sentidos $^{c}$ & Sistema nervioso $^{d}$ \\
\cline { 2 - 7 } Médicos & $3,1 \pm 1,1$ & $2,6 \pm 1,2$ & $3,6 \pm 1,2$ & $2,5 \pm 1,3$ & $2,6 \pm 1,3$ & $3,6 \pm 1,4$ \\
Alumnos & $3 \pm 1,2$ & $2,7 \pm 1,1$ & $3,3 \pm 1,2$ & $2,7 \pm 1,4$ & $3,4 \pm 1,4$ & $4,2 \pm 1$
\end{tabular}

Estudio según el cuadro que presenta el paciente

\begin{tabular}{cccccc|} 
& Cardiaco y respiratorio & Neurológico & Traumatológico & Ginecológico y obstétrico & Oftalmológico \\
\cline { 2 - 6 } Médicos & $3,7 \pm 1$ & $4,5 \pm 0,7$ & $4,7 \pm 1,2$ & $3,5 \pm 1,1$ & $3,4 \pm 1,3$ \\
Alumnos & $3,3 \pm 1,1$ & $4,4 \pm 0,9$ & $4,3 \pm 0,5$ & $3,6 \pm 1,3$ & $3,5 \pm 1,1$ \\
& Urológicos & Digestivos & Otorrinolaringológicos & Oncológicos & Dermatológicos \\
\cline { 2 - 6 } & $3,3 \pm 1,2$ & $3,9 \pm 1,3$ & $3,6 \pm 1$ & $2,9 \pm 1,1$ & $2,5 \pm 1,4$ \\
Médicos & $3,3 \pm 1,5$ & $3,6 \pm 1,1$ & $2,9 \pm 1,4$ & $2,2 \pm 1,2$ \\
Alumnos & $3,5 \pm 1,1$ & $3,4 \pm 1,2$ & 3,6 &
\end{tabular}

a La Bioquímica resultó mejor valorada como asignatura básica con aplicación clínica por los médicos en ejercicio que por los estudiantes $(P=0,01)$.

${ }^{\mathrm{b}}$ La Farmacología fue encontrada de mayor aplicación por los clínicos que por los estudiantes $(\mathrm{P}=0,001)$.

${ }^{c y d}$ Los estudiantes se han visto mas obligados a repasar sus conocimientos de órganos de los sentidos $(P=0,01)$ y de sistema nervioso $(P=0,03)$ para el estudio de las asignaturas clínicas, que los médicos para su ejercicio profesional.

TABLA 5. Definiciones y opiniones de los médicos de atención primaria y alumnos de sexto curso de la licenciatura en Medicina sobre la Anatomía Clínica

- Conocer las estructuras implicadas en los procesos clínicos mas frecuentes o aquellos infrecuentes de mayor gravedad.

- Estudio de las diferentes estructuras del cuerpo humano aplicadas al origen y desarrollo de enfermedades. Es básica en el ejercicio de la medicina.

- Relación de la Anatomía con el cuadro clínico que presenta el paciente indispensable para: localizar y denominar el lugar donde se localiza la patología.

- La aplicación de los conocimientos anatómicos a la patología. Es básica y necesaria

- Anatomía que ayuda a interpretar los signos y los síntomas que se presentan habitualmente en la práctica clínica.

- Es aquella que estudia los aspectos estructurales y funcionales (no meramente descriptivos del cuerpo humano) y que son mas útiles para la práctica médica, interpretación de técnicas de imagen y quirúrgicas. En ella se pone de manifiesto la anatomía del ser vivo y no exclusivamente del cadáver. Es la anatomía que precisa el generalista.

- Anatomía aplicada al diagnóstico. Es muy útil.

- Aplicación de los conocimientos anatómicos al diagnóstico y la terapéutica.

- Poder aplicar los conocimientos de anatomía a los signos/síntomas clínicos que expone el paciente. Es la aplicación a la consulta diaria y al ejercicio de la medicina.

- La Anatomía de la práctica diaria.

- El estudio de la Anatomía en relación con las alteraciones anatómicas que provocan las distintas patologías.

- Imprescindible

- Implicación de la Anatomía en la manifestación de la enfermedad.

- Fundamental en la consulta de atención primaria donde no disponemos de otras ayudas.

- Relación entre los síntomas clínicos y su localización, origen y posible causa.

- Muy Importante en la exploración de órganos y aparatos, en la inspección, palpación y auscultación. 
hubo diferencia estadísticamente significativa en la valoración de dichas actividades; exploración física $(P=0,99)$, interpretación de técnicas de imagen, $(P=0,2)$, interpretación de sintomatología $(P=0,5)$ y procedimientos terapéuticos $(P=0,69)$.

Tampoco hubo diferencia en la valoración de los diferentes aspectos de la Anatomía: descriptiva $(P=0,7)$, funcional $(P=0,6)$, imagen $(P=0,2)$, superficie $(P=0,1)$, topográfica $(P=0,1)$, y embriología $(P=0,6)$.

Alumnos y médicos coincidieron en que les había sido necesario repasar los conocimientos sobre el sistema nervioso y el aparato locomotor en primer y en segundo lugar respectivamente En menor medida los de órganos de los sentidos y sistema cardiovascular. No existió diferencia significativa entre la puntuación para cardio-vascular $(P=0,8)$, digestivo $(P=0,6)$, locomotor $(P=0,4)$ y uro-genital $(P=0,4)$. Sí existieron diferencias en la puntuación para órganos de los sentidos $(P=0,01)$ y sistema nervioso $(P=0,03)$, donde los al umnos manifestaron una mayor necesidad de repasar conocimientos.

Los datos no mostraron diferencia en la valoración, respecto a la necesidad de tener conocimientos anatómicos a la hora de estudiar un paciente con una patología determinada. Cardiológica y/o respiratoria $(P=0,06)$, dermatológica $(P=0,2)$, gastroenterológica $(P=0,4)$, neurológica $(P=0,3)$, obstétrica $(P=0,5)$, oftalmológica $(P=0,6)$, oncológica $(P=0,8)$, otorrinol aringológica $(P=0,07)$, traumatológica $(P=0,08)$ y urológica $(P=0,54)$.

\section{Opiniones sobre la docencia de la Anatomía Humana.}

Mientras que la gran mayoría delos profesionales considera que la ubicación de la Anatomía H umana en el curriaulum médico debería distribuirse en los dos ciclos (83\%), la opinión de los alumnos está dividida. El 41,6\% apoya la opción anterior, frente al $45 \%$ que opina que está bien ubicada en el primer cido. Médicos y alumnos, en su mayoría, modificarían la relación dase teórica / clase práctica con i ncremento de las últimas. Ambos grupos consideran precisa una visión mas aplicada de la anatomía (fig.3).

\section{DISCUSIÓ N}

Los resultados obtenidos suponen la primera aportación objetiva sobre las necesidades que en conocimientos de Anatomía Humana tienen los médicos de asistencia primaria y los alumnos del segundo cido de la titulación en Medicina de nuestra comunidad.
Curiosamente, las respuestas y comentarios en ambos grupos entrevistados fueron muy similares tanto en lo referente a la aplicación de conocimientos anatómicos, como en las necesidades de los mismos a la hora de la práctica dínica o de su utilización en las asignaturas dínicas del segundociclo. En estesentido los médicos de asistencia primaria y los alumnos de sexto curso opinaron que los conocimientos en A natomía Humana son fundamentales en la exploración física del paciente y en la interpretación de las técnicas de imagen, consideránddos menos relevantes a la hora de la inter pretación dela sintomatología y en los procedimientos terapéuticos.

Dado que el $87,5 \%$ de los facul tativos en atención primaria utiliza con asiduidad Rx simple y que la exploración física es una rutina diaria en asistencia primaria, los conocimientos anatómicos poseen de hecho una apl icabilidad elevada. Esto está en consonancia con la alta consideración dada a la anatomía de superficie y a la anatomía por la imagen por ambos grupos. Por otra parte, aunque a lo largo de la historia, anatomía y cirugía han estado estrechamente relacionados, la terapeútica fundamental mente farmacológica utilizada en los centros de salud explicaría la escasa asociación de los conocimientos anatómicos con los procedimi entos terapeúticos expresada por los médicos de atención primaria y la alta valoración atribuida por los estudiantes para el estudio de las asignaturas quirúrgicas.

Opinamos que también la sintomatología tiene en muchas ocasiones bases anatómicas capaces de explicarla. La baja valoración por parte de alumnos y profesionales clínicos en este sentido, la interpretamos como la necesidad de aplicar un enfoque adecuado en esta dirección a los conocimientos anatómicos que el profesor debe impartir. Este enfoque tendría como objetivo ayudar a que primero el alumno y posteriormente el médico sea capaz de interpretar la sintomatología con una base científica.

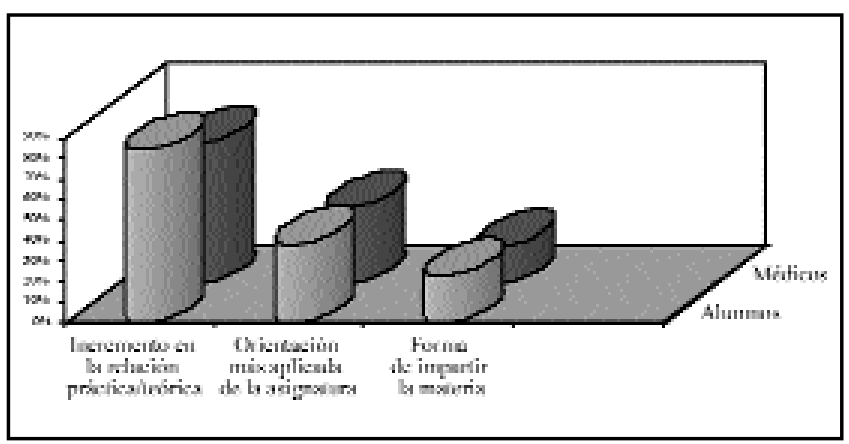

Fig.3: Modificarían en la Anatomía Humana que recibieron 
Es interesante observar la coincidencia de que sean los contenidos de sistema nervioso y aparato locomotor los considerados de mayor importancia a la hora de su aplicación y los que necesitan ser repasados con mayor asiduidad, tanto por los profesionales médicos en su práctica diaria, como por los alumnos para el estudio de las asignaturas dínicas. Nos queda, sin embargo, sin aclarar si es consecuencia de: 1. una mayor incidencia de procesos patológicos que afecten al aparato locomotor y al sistema nervioso, 2. si las bases anatómicas de los mismos están en estrecha relación con su sintomatología y la exploración de estos aparatos o 3. si por el contrario lo que ocurre es que estas partes de la anatomía poseen contenidos extensos y probablemente mas difíciles de recordar. En cualquier caso es un tema a tener en cuenta a la hora de la preparación de los programas y distribución de horas de los diferentes contenidos de los mismos.

La embriología fue considerada de muy poco interés tanto en el estudio de las asignaturas clínicas como en su utilidad en asistencia primaria. Como profesores de Anatomía, la embriología nos resulta interesante para comprender la forma anatómica definitiva, para explicar determinadas asociaciones dínicas, así como para el estudio de las malformaciones. Los resultados nos Ilevan a cuestionarnos si, no sería necesaria una integración mucho más estrecha de la embriología con la descripción morfológica definitiva de una estructura determinada y con el estudio de los cuadros clínicos de malformaciones en las asignaturas a las que correspondan, para que la embriología fuese útil.

Por otra parte, resulta evidente que no solamente la disciplina en sí, sino también las tendencias docentes del profesorado que imparte la asignatura, pueden influir en la capacidad del futuro profesional a la hora de aplicar los conocimientos adquiridos. Este hecho es corroborado por las diferencias en valoración sobre distintos aspectos de la Anatomía Humana que fueron expresadas por los médicos según el año de finalización de la licenciatura.

Respecto a su papel en el curríaulum médico, los dos grupos consideraron la Anatomía Humana entre lastres asignaturas básicas más valorada a la hora de la aplicación clínica. Pero, mientras que los alumnos la situaron en segundo lugar después dela Fisiología los médicos de asistencia primaria lo hicieron en tercero. La Farmacología del tercer lugar atribuido por los alumnos, pasó al primero considerado por los dínicos. Este hecho corrobora, desde nuestro punto de vista, la importancia quela terapéutica fundamental- mentefarmacológica tiene hoy en asistencia primaria. Creemos que este es un dato adicional de gran trascendencia a tener en auenta no sdo por los docentes, sino también por las instituciones sanitarias.

En consonancia con las tendencias de defensores de la Anatomía clínica9, los médicos de asistencia primaria entrevistados, consideraron en su mayoría que sería de interés incluir en el currículum médico una Anatomía general durante el primer ciclo de la Licenciatura así como una Anatomía Humana aplicada durante el segundo cido una vez que el alumno ya tuviese nociones de patología. Casi la mitad de los alumnos consideraron interesante esta posibilidad. Lo anterior está en correspondencia con las opiniones de alumnos de la licenciatura en Medicina recogidas en otros trabajos?. La defensa de esta ubicación por los anatómicos clínicos se basa fundamentalmente en dos hechos, 1. En los últimos cursos el alumno ha olvidado probablemente la anatomía básica requerida para comprender la patología relevante y 2 . Es prácticamente imposible memorizar la anatomía humana a lo largo de toda la vida profesional cuando esta ha sido estudiada solo en los primeros años de la licenciatura?.

Alumnos y profesionales en ejercicio consideraron que la Anatomía Humana debería ser una asignatura fundamentalmente práctica, donde no solo la disección sino las técnicas de imagen y la resolución de problemas clínicos con base anatómica deberían ocupar un lugar fundamental.

Podemos conduir que el concepto clásico de que la anatomía macroscópica es uno de los pilares de la Medicina permanece vigente a pesar de las opiniones en contra vertidas en los últimos años y las reducciones a las que se ha sometido la disciplina en el curriculum médico en la mayoría de los países. Nuestro trabajo nos muestra, sin embargo, la opinión prácticamente generalizada de que se hace preciso dirigir la docencia de la Anatomía Humana hacia el estudio del ser humano vivo y la práctica dínica. Para ello consideramos que es más necesario realizar modificaciones en la metodología y orientación docente, que hacer grandes variaciones en los contenidos. Como ya ha sido sugerido por otros autores, más anatomía de superficie, más anatomía del ser humano vivo, orientada en todos los casos hacia la profesión médica ${ }^{10,11}$.

\section{AGRADECIMIENTOS}

Este estudio ha sido financiado por el programa propio de ayudas a la investigación de la Universi- 
dad de Las Palmas de Gran Canaria (1999). Damos las gracias al Servicio Canario de Salud y a su director médico Dr. Marrero, así como a los profesionales y estudiantes por su colaboración en la realización de este proyecto.

De forma especial nos gustaría agradecer las facilidades que nos brindaron los coordinadores de los Centros de Salud de: J inamar: Dr. Dámaso Campos, Barrio Atlántico: Dra. Juana Santana, Canalejas: J osé Ma Ortega, Firgas: Dra. Anabel Delgado, y Escaleritas: Dr. Ignacio Martín.

\section{BIBLIOGRAFÍA}

1. Guilbert J J. . Guía Pedagógica para el personal de la Salud. Sexta Edición Ed: Universidad de Valladolid, 1994.

2. Chevrel J P. Clinical anatomy: the new spirit in anatomy Surg. Radiol. Anat. 1992;14:93-95.

3. O.M.S. Introducción a la preparación de planes de estudio basados en la competencia. Cuadernos de Salud Pública 1978;68:23.

4. Baker MZ.,Scoffield RH. Educational needs of internal medicina residency graduates:general internist versus subspecialists.Med Educ 1998;32:527-532.
5. Manso Martinez J .M. (2000) ¿Qué enseñar en ciencias de la salud? Técnicas para definir compe tencias y perfiles profesionales (y II) Educación Médica 3:101-106.

6. Strate J. Rothkotter HJ ., Pabst R. How do medical students judge preclinical studies? Results of questionnaire analysis after the 1st and 2nd year of studies. Dtsch. Med. Wochenschr. 1998; 123:1093-6.

7. Pabst R. Gross anatomy: an outdated subject or an essential part of a modern medical curriculum? Results of a questionnaire circulated to final-year medical students. Anat. Rec. 1993; 237:431-3.

8. Pabst R., Rothkotter HJ . Retrospective evaluation of undergraduate medical education by doctors at the end of their residency time in hospitals: consequences for the anatomical curriculum. Anat. Rec 1997;249:431-4.

9. Chevrel J P The modern teaching of modern anatomy. Surg. Radiol. Anat. 1995 17:285-6.

10. Cahill Dr., and SW. Carmichael Supplemental dinical dissections for freshman gross anatomy. Anat.Rec. 1985;212:218-222.

11. Monkhouse WS. Anatomy and the medical school curriculum. Lancet 1992;340:834-835.

\section{ANEXO 1: Cuestionario para los médicos de asistencia primaria}

1. Lugar de trabajo.

Centro de Salud ...........

2. Situación laboral:

a. Titular de la plaza b. Contratado (interino) c. Contratado (sustituto)

d. Residente MIR

3. Sexo

a. Varón b. Mujer

4. Su titulación actual es:

$a$. Médico generalista $b$. Especialista en Medicina familiar y comunitaria.

c. Otra especialidad (especificar)...

5. Año en el que finalizó su licenciatura:

a. Tres últimos años b. Entre 1990-1995 c. Entre 1985-1989

d. Entre 1980-1984 e. Con anterioridad a 1980

6. Respecto a las siguientes disciplinas básicas. Valore de $\mathbf{1}$ a 5 (1 poca, 5 mucha) la importancia que para su actividad diaria tiene cada una de ellas.

a. Bioquímica b. Anatomía Humana c. Citología e Histología

d. Fisiología e. Farmacología

7. Valore de 1 a 5 ( 1 poca, 5 mucha) la relevancia que tienen los conocimientos anatómicos en:

a. Exploración física $b$. Interpretación de técnicas de imagen

$c$. Interpretación de la sintomatología $d$. Procedimientos terapéuticos

8. Valore de 1 a 5 ( 1 poca, 5 mucha)la importancia que usted concede a:

a. Anatomía de superficie. b. Anatomía descriptiva. c. Anatomía topográfica

d. Anatomía por la imagen e. Embriología $f$. Anatomía funcional.
9. ¿Cuál es la frecuencia con la que utiliza las siguientes técnicas diagnósticas? Frecuentemente (4), A veces (3), Casi nunca (2), Nunca (1)

a. Radiología simple $b$. Radiología con contraste.

c. Tomografías computarizadas. $d$. Resonancias magnéticas.

e. Ecografías.

10. ¿Qué valoración atribuye dentro de los programas de Anatomía Humana a la anatomía por la imagen?.

a. Debería ocupar un lugar muy importante

b. Debería ocupar un lugar importante

c. Serían necesarias solo unas nociones

d. No tiene sentido incluirla en los programas de Anatomía Humana

e. Otros(especificar)..........

11. ¿Qué opina de la Anatomía de superficie?

$a$. Es poco útil $b$. Es necesaria $c$. Es fundamental

d. No sirve para nada e. Otros(especificar)............

12. Haga una valoración de los conocimientos anatómicos que conserva de su etapa de estudiante.

$\begin{array}{llll}\text { a. }>75 \% & \text { b. } 50-75 \% & \text { c. } 25-50 \% & \text { d. }<25 \%\end{array}$

13. Durante su ejercicio profesional se ha visto obligado a repasar conocimientos de Anatomía Humana.

a. Una vez al mes $b$. Una vez cada 6 meses $c$. Una vez al año d. Una vez cada 2 o 3 años e. Nunca $f$. Otros (especificar)...........

14. Durante su actividad profesional ha precisado repasar sus conocimientos anatómicos de.... Puntúe de 1 a 5 (1 poco, 5 mucho) a. Sistema nervioso. b. Aparato locomotor

c. Aparato cardio-vascular y respiratorio $d$. Aparato digestivo

$e$. Uro-genital $f$. Órganos de los sentidos g. Otros. 
15. Puntúe de 1 a 5 (1= poco importante, $5=$ muy importante) la necesidad de conocer las bases anatómicas a la hora de estudiar un paciente con problemas:

a. Neurológicos b. Traumatológicos c. Cardiovasculares y/o respiratorioss d. Gastroenterológicos e. Urológicos $f$. Ginecológicos-Obstétricos

g. Otorrino-Laringológicos $h$. Oftalmológicos i. Dermatológicos

j. Oncológicos k. Otros.

16. Basándose en su experiencia, ¿Qué opina sobre el hecho de que la Anatomía Humana como disciplina se imparta únicamente durante el primer ciclo de la licenciatura?

a. Está muy bien $b$. La Impartiría durante el segundo ciclo.

c. Impartiría una Anatomía Humana general durante el primer ciclo y una Anatomía aplicada durante el segundo ciclo.

d. Eliminaría la Anatomía Humana de los planes de Estudio de la Licenciatura.

e. Disminuiría la cantidad de horas dedicadas a la disciplina. $f$. Otras......

17. Si tuviese que elaborar un programa de Anatomía Humana que proporción del tiempo dedicaría a:

a. Lecciones magistrales $b$. Disección $c$. Estudio de piezas de cadáver d. Técnicas de imagen e. Resolución de problemas con orientación clínica $f$. Estudio de maquetas $g$. Libros para colorear, sistemas multimedia

h. Anatomía de superficie i. Otros (especificar)

18. ¿Qué objetivos deberían alcanzar los alumnos durante el aprendizaje de la Anatomía Humana en la Licenciatura en Medicina?
19. ¿Qué es para usted la anatomía Clínica?, ¿Qué opina de la misma?

20. ¿Qué modificaría y que conservaría de la enseñanza de la Anatomía Humana que usted recibió?

a. Estoy muy conforme con la misma

b. Estoy totalmente disconforme con la misma

c. Sugeriría modificaciones........... (especificar)

21. ¿Considera interesante la realización de cursos de postgrado sobre bases anatómicas de diferentes actividades clínicas.

a. Si b. No c. Otros (especificar)

22. Enumere 5 procedimientos diagnósticos y/o terapéuticos que utilice durante un día de actividad normal y que estén basados en conocimientos anatómicos.

23. ¿Estaría dispuesto a participar en una evaluación de sus conocimientos anatómicos?

a. Si, directamente b. Si, tras un curso de actualización $c$. No d. Otros (especificar)

24. ¿Estaría dispuesto a participar en grupos de trabajo dirigidos a la elaboración de objetivos educativos?

a. Si b. No c. Otros (especificar)

\section{ANEXO 2: Cuestionario para los alumnos}

\section{Sexo}

a. Varón b. Mujer

2. Curso de comienzo de la licenciatura

$\begin{array}{lllll}\text { a. } 93 / 94 & \text { b. } 92 / 93 & \text { c. } 91 / 92 & \text { d. } 90 / 91 & \text { e. Con anterioridad a } 1990\end{array}$

3. Haz una valoración de 1 a 5 , de cada una de las siguientes asignaturas básicas según la relación que tengan con las asignaturas clínicas.

a. Anatomía Humana b. Bioquímica $c$. Citología e histología

d. Farmacología e. Fisiología

4. Haz una valoración de 1 a 5 según la importancia que para ti tienen los conocimientos anatómicos en:

$a$. Exploración física $b$. Interpretación de técnicas de imagen

c. Interpretación de la sintomatología $d$. Procedimientos terapéuticos.

5. Valora de 1 a 5 la relevancia para la clínica de:

$a$. Anatomía de superficie $b$. Anatomía descriptiva $c$. Anatomía topográfica

d. Anatomía por la imagen e. Embriología f. Anatomía funcional.

\section{6. ¿Qué valoración atribuyes tú dentro de los programas} de Anatomía Humana a la Anatomía por la imagen?

a. Debería ocupar un lugar importante $b$. Sería necesario solo unas nociones.

c. No tiene sentido incluirla en los programas de Anatomía. d. Otros

7. ¿Qué opinas de la Anatomía de superficie?

$a$. Es poco útil $b$. Es necesaria $c$. Esa fundamental $d$. No sirve para nada

e. Otros.

8. Haz una valoración de los conocimientos anatómicos que conservas de cuando estudiaste la asignatura

$\begin{array}{llll}\text { a. }>75 \% & \text { b. } 50-75 \% & \text { c. } 25-50 \% & \text { d. }<25 \%\end{array}$

9. Durante el estudio de las asignaturas clínicas te has visto obligado a repasar conocimientos anatómicos

$a$. Una vez al mes $b$. Una vez cada seis meses $c$. Una vez al año

$d$. Una vez cada dos años $e$. Nunca $f$. Otros...

10. Durante el estudio de las asignaturas clínicas he precisado repasar los contenidos anatómicos de (puntúa de 1 a 5).

a. Sistema nervioso $b$. Aparato locomotor

c. Aparato cardiovascular y respiratorio $d$. Aparato digestivo

$e$. Urogenital $f$. Órganos de los sentidos. $g$. Otros.........

11. Puntúa de $\mathbf{1}$ a 5 la necesidad de conocer las bases anatómicas a la hora de estudiar un paciente con problemas: a. Neurológicos b. Traumatológicos $c$. Cardiovasculares

d. Gastroenterológicos e. Urológicos f. Obstétricos y ginecológicos

$g$. Otorrinolaringológicos $h$. Oftalmológicos $i$. Dermatológicos

j. Oncológicos $k$. Otros

12.Puntúa de 1 a 5 la necesidad que has tenido de conocimientos anatómicos en el estudio de:

a. Patología general $b$. Radiología general $c$. Fundamentos de cirugía

d. Oftalmología e. Medicina de Urgencias $f$. Otorrinolaringología

h. Prácticas clínicas

\section{3.¿Qué opinas sobre el hecho de que la Anatomía Humana sea} impartida durante el primer ciclo de la licenciatura.

a. Está muy bien b. Estaría mejor en segundo ciclo

c. Sería mas provechoso yuna Anatomía Humana general durante

el primer ciclo y una aplicada durante el segundo.

d. Eliminaría la Anatomía Humana de los planes de Estudio.

e. Disminuiría la cantidad de horas dedicadas a la disciplina $f$. Otras.

14. Si tuvieses que elaborar un programa de Anatomía Humana. ¿Qué proporción del tiempo dedicarías a?

a. Lecciones magistrales. b. Disección $c$. Técnicas de imagen

d. Resolución de problemas con orientación clínica.

$e$. Estudio de maquetas $f$. Sistemas de auto-aprendizaje (libros para

colorear, multimedia etc) g. Anatomía de superficie $h$. Otras (especificar)......

15. ¿Qué aptitudes y que destrezas consideras que debe desarrollar el alumno de Anatomía Humana durante su aprendizaje?

16. ¿Qué es para ti la anatomía clínica?, ¿Qué opinas de la misma?

17. ¿Qué modificarías y que conservarías de la enseñanza de la anatomía que recibiste.

18. ¿Consideras interesante la realización de cursos de post-grado de bases anatómicas de diferentes actividades clínicas? a. Si b. No c. Otros (especificar)

19. Enumera 5 procedimientos diagnósticos o terapéuticos que estén basados en conocimientos anatómicos.

a. b. c. d. e.

20.¿Estarías dispuesto a participar en una evaluación de tus conocimientos anatómicos actuales?

a. Si b. No c. Otros. 